\title{
Bases teóricas, aplicación, operacionalización y medición de las variables en psicología
}

\section{Theoretical bases, application, operationalization and measurement of variables in psychology}

\author{
Karina Reyes-Jarquín ${ }^{a}$, M. Leticia Bautista-Díaz ${ }^{b}$, Angélica Romero-Palencia ${ }^{c}$, Ana Luisa \\ Mónica González-Celis Rangel ${ }^{d}$
}

\begin{abstract}
:
Variables are central elements in scientific activity, including psychology, however, it can be complex to identify their function and application. The present work aims to provide an overview of the variables from research in the psychological field, delves into the definition of concept, construct and variable; in addition to explaining and exemplifying five classifications of the variables; I) by the number of its securities; II) by its nature; III) by the level of measurement; IV) by the degree of manipulation; V) by its definition; Likewise, it allows us to deepen the importance of the conceptual and operational definition, favoring the understanding of the congruence between the theoretical and empirical level of the variables. Finally, it is concluded that the clarity that a study manifests in its variables is a key point for its planning, execution and communication of results.
\end{abstract}

Keywords:

Research methodology, variable, construct, instrument, measurement

\section{Resumen:}

Las variables son elementos centrales en la actividad científica, incluida la psicología, no obstante, puede ser complejo identificar su función y aplicación. El presente trabajo tiene como finalidad brindar un panorama respecto a las variables desde la investigación en el ámbito psicológico, se profundiza en la definición de concepto, constructo y variable; además de explicar y ejemplificar cinco clasificaciones de las variables; I) por el número de sus valores; II) por su naturaleza; III) por el nivel de medición; IV) por el grado de manipulación; V) por su definición; asimismo, permite profundizar en la importancia de la definición conceptual y operacional favoreciendo la comprensión de la congruencia entre el nivel teórico y empírico de las variables. Finalmente, se concluye que la claridad que un estudio manifieste en sus variables es un punto clave para su planeación, ejecución y comunicación de resultados.

Palabras Clave:

Metodología de la investigación, variable, constructo, instrumento, medición

\section{Introducción}

Cuando se habla de investigación se toman en cuenta dos niveles importantes del proceso: el teórico y el empírico. El nivel teórico tiene que ver con el planteamiento de un problema de investigación, las teorías y la hipótesis, mientras que el empírico tiene que ver con observar, medir o evaluar para comprobar la hipótesis y concluir con una teoría, regresando así a un nivel teórico.

\footnotetext{
Autor de Correspondencia, Universidad Nacional Autónoma de México, https://orcid.org/0000-0003-3660-3594, Email: krjarquin@gmail.com

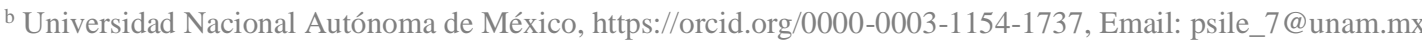

c Universidad la Salle Pachuca, https://orcid.org/0000-0003-0933-6937, Email: aacrom@gmail.com

d Universidad Nacional Autónoma de México, https://orcid.org/0002-8996-5866, Email: algcr10@ hotmail.com
} 
Si se plantea: "el estrés, produce ansiedad", esta oración sería una hipótesis integrada por dos elementos "estrés" y "ansiedad" que van unidos por un verbo "produce". Esta hipótesis pertenece al nivel teórico.

De acuerdo con el método científico, no sería correcto mantenerse sólo en este nivel ya que el planteamiento debe llegar al segundo. Para hacerlo se debe pasar a acciones como la observación, medición o evaluación de los elementos involucrados en la hipótesis. Pero ¿se puede observar, medir o evaluar el "estrés" y la "ansiedad"? Para responder a la pregunta, primero se deben definir sus elementos de tal modo que sea posible observarlos o evaluarlos indirectamente.

\section{Concepto, constructo y variable.}

De acuerdo con Kerlinger y Lee ${ }^{1}$ los términos "concepto", "constructo" y "variable" tienen significados similares, aunque existen diferencias importantes entre ellos, como se verá a continuación.

La Real Academia Española de la Lengua ${ }^{2}$ indica que la palabra "concepto" proviene del latín conceptus y es una representación mental creada por una generalización. Moulines $^{3}$ afirma que es la unidad más básica de toda forma de conocimiento humano. Un concepto, habitualmente obtiene su nombre a partir del consenso y se emplea para fines prácticos. Hay conceptos que son concretos y fáciles de observar como "mesa", "libro", "animal" y otros abstractos como "clima", "sentimiento "o "masa". Los seres humanos en general emplean estos conceptos y los utilizan todo el tiempo para comunicarse.

Por el contrario, Kerlinger y Lee $^{1}$ indican que "un constructo es un concepto que ha sido adaptado o creado para un propósito científico, de forma deliberada y consciente" (p. 36). Es decir, los constructos son los conceptos de la ciencia que se obtienen a partir de la observación científica de los comportamientos o las características que los conforman, pero no se pueden observar directamente.

Por ejemplo, en una investigación se le llama "depresión" al conjunto de características como: dificultad para conciliar el sueño o exceso de sueño, cambio en el apetito, cansancio y falta de energía, sentimientos de inutilidad, falta de concentración, retraimiento de las actividades usuales y sentimientos de desesperanza o abandono ${ }^{4}$. Así, depresión es un constructo que se obtendrá a partir de la observación de estas características cuando se presenten en conjunto.
En investigación de manera consciente y sistemática se usan los constructos en dos formas: 1) incluyéndolos en hipótesis o teorías y relacionándolos con otros y 2) definiéndolos claramente, de tal forma que se puedan observar, medir o evaluar.

En el ámbito científico en general, a los constructos o propiedades que se estudian también se les puede denominar "variables", principalmente cuando son representadas por letras, regularmente las últimas del abecedario como $\mathrm{x}, \mathrm{y}, \mathrm{z}$, -aunque en algunas ciencias como la psicología no se les suele colocar letra-, si no que se conceptualizan y operacionalizan.

Por definición "una variable es una propiedad que asume diferentes valores"1 (p. 36) o "una característica observable de algo que puede fluctuar y cuya variación es

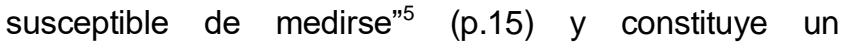
componente primordial del desarrollo de toda investigación.

Para Ritchey ${ }^{6}$ (p. 10) "una variable es un fenómeno medible que varía o cambia a través del tiempo, o que difiere de un lugar a otro o de un individuo a otro. Así, las variables son las características de los sujetos u objetos bajo estudio". De acuerdo con Cruz, Olivares y González ${ }^{7}$ cuatro propiedades de las variables son: a) sustantivas, b) cambiantes, c) multivalor, y d) definibles.

Así, las variables son lo opuesto de las constantes (generalmente representadas por las primeras letras del alfabeto como a, b, c), ya que en el caso de estas últimas como su nombre lo indica solo pueden adoptar un valor y este nunca cambia, un ejemplo muy común es el símbolo $\pi(p h i)$ que en términos generales se reconoce con el valor de 3.1416 que se emplea para varios cálculos matemáticos, ese valor nunca cambia.

Como se mencionó anteriormente, en la esfera científica, una variable se representa generalmente con una de las últimas letras del abecedario, a la que se le asignan valores, categorías o números. Por ejemplo, si $X$ es la variable en estudio, en este caso, puntajes en una escala de ansiedad, se asigna a $X$ un conjunto de valores numéricos proporcionados por la escala de ansiedad que se esté empleando. Este grupo de valores podría variar por ejemplo de 0 a 170. Pero en otro estudio una variable $X$, puede tener sólo dos valores. Por ejemplo, si el sexo es el constructo bajo estudio, entonces a $X$ se le pueden asignar valores de 1 y 2 , donde 1 representa uno de los sexos y 2 el otro.

En el caso específico de la Psicología, todos los elementos que conforman el proceso de la actividad 
científica, desde la planeación, ejecución e informe de un protocolo de investigación cuantitativa, consideran el estudio de variables, es decir, estas deben estar presentes en el marco teórico, planteamiento del problema, método, resultados, discusión, conclusión, limitaciones del estudio y las futuras líneas de investigación..$^{1,7,8}$ En síntesis, las variables son las que se conceptualizan, operacionalizan, cuantifican, miden, estudian, manipulan o controlan, con el propósito final de comunicarlas a la comunidad académica y científica.

La revisión bibliográfica acerca de un tema o la observación del fenómeno pueden ayudar a familiarizarse con el tipo de variables insertas en el tema de interés a investigar. ${ }^{5}$ Por lo tanto, antes de iniciar el nivel empírico de la investigación es importante pasar por el nivel teórico, revisando literatura, eligiendo cuidadosamente las variables y definiéndolas de tal forma que se puedan observar o medir y conociendo las propiedades, cualidades y alcances de las variables, para llevar adecuadamente el proceso de investigación, por lo que es necesario conocer su clasificación.

\section{Clasificación de las variables}

Las variables pueden clasificarse de acuerdo con diversas características, tales como el número de valores, la naturaleza, el nivel de medición, el grado de manipulación, entre otros. A continuación, se especifica dicha clasificación.

\subsection{Clasificación por el número de valores.}

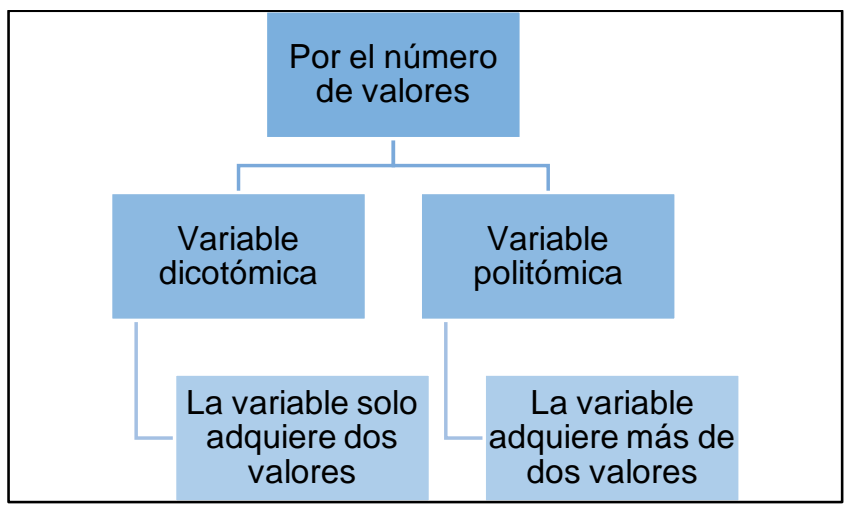

Fig. 1. Clasificación de las variables por el número de valores

Como se observa en la Figura 1, esta clasificación depende del número de valores que adopte cada variable, al tener sólo dos valores se nombra variable dicotómica; mientras que, si existen tres o más valores se nombra como variable politómica. ${ }^{9}$

En las siguientes oraciones se ejemplifican algunas variables dicotómicas y politómicas:

1. Las personas sanas obtuvieron puntajes más altos en Calidad de vida, al ser comparadas con personas enfermas.

La variable estado de salud se clasificaría como dicotómica cuando los valores que contiene son 2: salud y enfermedad.

2. En un grupo de amas de casa, $40 \%$ reportó estar satisfecha con la vida, mientras $60 \%$ restante indicó estar insatisfecha con la vida.

La variable satisfacción con la vida se clasificaría como dicotómica cuando los valores que contiene son 2: satisfacción e insatisfacción.

3. Los cuidadores informales que recibieron una intervención breve cognitivo conductual en una institución dedicada a la atención del adulto mayor, reportaron ausencia de sobrecarga del cuidador; en comparación con los cuidadores que no recibieron dicha intervención y reportaron presencia de sobrecarga.

La variable sobrecarga del cuidador se clasificaría como dicotómica cuando los valores que contiene son 2: presencia y ausencia.

4. Los participantes con un nivel superior de escolaridad (preparatoria o licenciatura) reportaron mayor autoeficacia en comparación con quienes tenían un nivel más bajo de escolaridad (primaria o secundaria).

La variable escolaridad se clasificaría como politómica debido a que tiene más de dos valores, en este caso 4: primaria, secundaria, preparatoria y licenciatura.

5. Los participantes con un nivel alto de calidad de vida también presentaron un mayor bienestar psicológico, en comparación con quienes indicaron un nivel de calidad de vida bajo $o$ medio.

La variable nivel de calidad de vida se clasificaría como politómica debido a que tiene más de dos valores, en este caso 3: nivel bajo, nivel medio y nivel alto. 
6. Los estudiantes que empleaban estrategias de afrontamiento como apoyo social y expresión emocional reportaron menor nivel de estrés, en comparación con los estudiantes que emplean estrategias de afrontamiento tales como evitación de problemas, pensamiento desiderativo, retirada social y autocrítica.

La variable estrategias de afrontamiento se clasificaría como politómica debido a que tiene más de dos valores, en este caso 6: apoyo social, expresión emocional, evitación de problemas, pensamiento desiderativo, retirada social y autocrítica.

\subsection{Clasificación por su naturaleza}

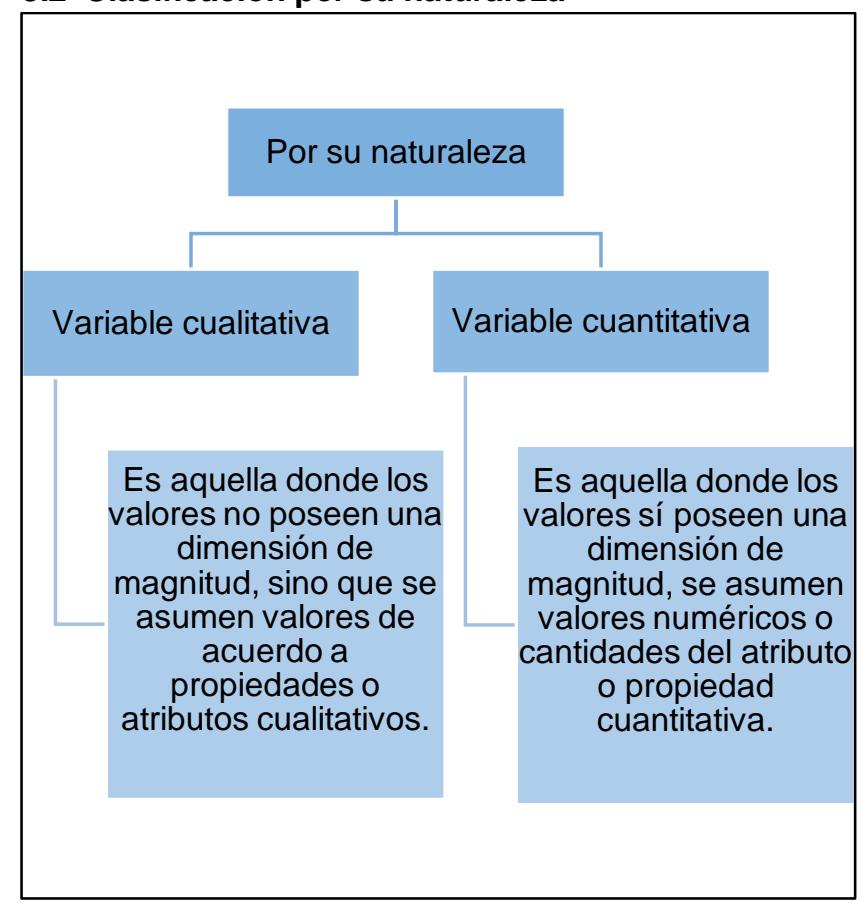

Fig. 2. Clasificación de las variables por su naturaleza

Como se observa en la figura 2 , esta clasificación depende de la naturaleza de la variable; si las propiedades no poseen una dimensión de magnitud se hablará de una variable cualitativa; $y$, por el contrario, si las propiedades poseen una dimensión de magnitud se hablará de una variable cuantitativa. ${ }^{10}$

En las siguientes oraciones se ejemplifican algunas variables cualitativas y cuantitativas:

1. Las profesoras de una preparatoria reportaron mayores niveles de ansiedad al ser comparadas con los profesores de la misma institución.

La variable género se clasificaría como cualitativa dado que sus valores (masculino y femenino) no poseen una dimensión de magnitud; es decir, no existe una diferencia numérica entre ambos valores. Se pueden emplear símbolos para diferenciarlos, por ejemplo, asignar la letra $M$ para identificar el género masculino, y la letra $F$ para el género femenino; o incluso el numeral 1 para el género masculino y el numeral 2 para el género femenino sin que esto implique que exista una diferencia numérica entre ambos valores.

2. Los cuidadores informales desarrollan un lazo afectivo con la persona a la que cuidan, a diferencia de los cuidadores formales.

La variable tipo de cuidador se clasificaría como cualitativa, dado que sus valores (cuidador formal y cuidador informal) no poseen una dimensión de magnitud. Se pueden emplear símbolos para diferenciar ambos valores, por ejemplo, asignar la letra $\mathrm{F}$ para identificar a los cuidadores formales, y la letra I para los cuidadores informales; o incluso algún numeral como el 5 para los cuidadores formales, y el numeral 8 para los cuidadores informales, sin que esto implique que exista una diferencia aritmética entre ambos.

3. Los participantes de acuerdo con su edad presentan diferentes características de deterioro relacionadas con el envejecimiento. De esta manera los participantes de 18 años tendrán menor deterioro que aquellos con 58 años.

La variable edad se clasificaría como cuantitativa dado que sus valores ( 10 años, 18 años, 34 años, 58 años, etc.) sí poseen una dimensión de magnitud existiendo una diferencia numérica entre los valores de dicha variable. Los números que distinguen cada uno de los valores, implican diferencia aritmética entre ellos; un valor más pequeño representa una menor edad, y un valor más grande una edad mayor.

4. En una prueba de funciones ejecutivas en adultos mayores, el paciente que terminó más rápido (en 9 minutos) obtuvo un puntaje más bajo que aquellos que tardaron más (13 y 15 minutos).

La variable tiempo de ejecución en una prueba de funciones ejecutivas se clasificaría como cuantitativa dado que sus valores (9 minutos, 13 minutos, 15.5 minutos) sí poseen una dimensión de magnitud existiendo una diferencia numérica entre los valores.

\subsubsection{Clasificación de las variables cuantitativas}

Específicamente en las variables cuantitativas existe una subclasificación como se observa en la figura 3 . 


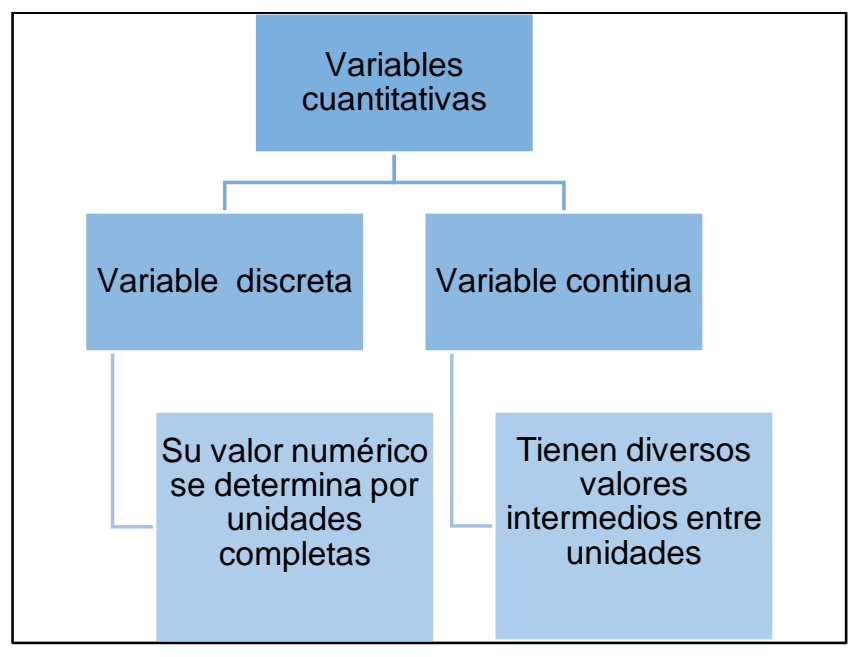

Fig. 3. Clasificación de variables cuantitativas

En el caso de las variables discretas, se trata de variables cuyo valor numérico se determina por unidades completas, es decir solo pueden adquirir valores enteros. Mientras que, las variables continuas se caracterizan por tener diversos valores intermedios entre unidades, por lo tanto, pueden adquirir valores fraccionarios. A continuación, se presentan algunos ejemplos.

1. En una tarea realizada en la Torre de Londres, una niña de seis años con daño neurológico presentó diez movimientos para imitar un modelo, mientras que un niño de siete años presentó 13 movimientos.

En el ejemplo anterior, los valores de la variable movimientos se encuentran en unidades completas (seis o 13), por tanto, es una variable discreta.

2. Un psicólogo de la salud midió el número de errores en el seguimiento de instrucciones $y$ encontró que un niño de 6 años presentó cuatro errores, mientras que uno de 8 presentó seis.

En el ejemplo anterior el número de errores se presenta en unidades completas (cuatro y ocho), de este modo esta variable es discreta.

3. En una tarea realizada en la Torre de Londres, una niña de seis años con daño neurológico tuvo una latencia de 3.45 minutos en la resolución de una tarea.

En este caso, el valor de la latencia es continua, ya que entre el minuto 3 y el 4 hay 45 segundos mediando estas unidades, así puede haber valores continuos entre la unidad previa y la posterior.
4. En un estudio sobre reducción de peso, se registró en una paciente el peso de $110.56 \mathrm{k}$. antes de la cirugía bariátrica.

En este caso el peso corporal es una variable continua, ya que, entre 110 y $111 \mathrm{k}$ hay un número importante de fracciones, estos puedes ser gramos en centésimas o décimas.

\subsection{Clasificación por el nivel de medición}

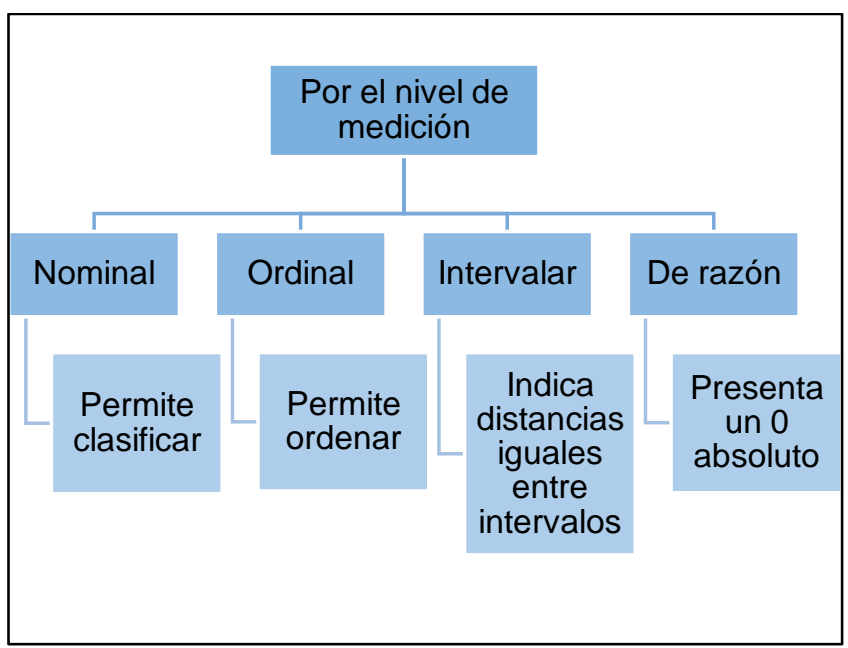

Fig. 4. Clasificación de las variables por el nivel de medición

Para hablar de la clasificación por nivel de medición de la variable es necesario hacer un breve repaso de las características esenciales de cada nivel de medición. ${ }^{10,11}$

Nivel de medición nominal

a) Es la forma más simple de medición.

b) Implica la asignación o clasificación en categorías mutuamente excluyentes.

c) No indica ningún orden en particular ni magnitud entre los valores.

d) Se asignan símbolos o numerales que carecen de un significado numérico.

e) Sólo se establece si aquello que se está midiendo es igual o diferente de las categorías determinadas.

Nivel de medición ordinal

a) Incluye un ordenamiento de menor a mayor o viceversa por rangos con base en alguna característica.

b) No permite operaciones aritméticas.

c) Se cumple con el postulado de transitividad: si "a" es mayor que "b", y "b" es mayor que "c", entonces "a" es mayor que "c". 
d) La distancia entre los valores de la escala sólo representa orden, pero no magnitud o distancia igual para cada punto de la escala.

e) Se asignan numerales que reflejan el orden en la escala.

Nivel de medición intervalar

a) Señala el grado de diferencia entre dos puntuaciones, estableciendo que existan intervalos iguales.

b) La distancia entre intervalos es la misma a lo largo de toda la escala.

c) Contiene un 0 arbitrario, no real; es decir, se asigna un punto de partida arbitrario desde donde se construye o inicia la medición.

d) Se permiten operaciones aritméticas.

Nivel de medición de razón

a) Considera las propiedades de los niveles de medición anteriores y contiene un 0 absoluto, lo que implica que hay un punto en la escala donde está ausente la propiedad medida.

En las siguientes oraciones se ejemplifican las variables de acuerdo a su nivel de medición:

1. En un grupo de personas con diabetes, aquellas que no tomaban sus medicamentos en los horarios correspondientes tuvieron niveles de glucosa mucho mayores que aquellas personas que tomaban sus medicamentos en el horario indicado.

La variable adherencia al tratamiento medida en personas con diabetes mediante la pregunta ¿Toma su medicamento en los horarios correspondientes? Sí / No; se clasificaría como una variable nominal, debido a que se establecen dos categorías mutuamente excluyentes, aquellos que toman su medicamento en los horarios correspondientes y aquellos que no lo hacen. A quienes respondan que sí toman sus medicamentos en los horarios establecidos se les puede identificar con el numeral $1 \mathrm{y}$ al resto con el numeral 2, sin que esto signifique que exista una diferencia aritmética.

\section{Se observó en un grupo de pacientes hipertensos} que entre mayor era su nivel de bienestar psicológico (nivel alto, medio y bajo) menor era su nivel de estrés.

Se clasificaría como variable ordinal al bienestar psicológico evaluado mediante un instrumento creado ex profeso, que arroja 1 de 3 posibles niveles: alto, medio y bajo. Lo anterior se debe a que se forman tres categorías mutuamente excluyentes pero que sí pueden ordenarse de menor a mayor o viceversa.
3. Los adultos mayores que reportaron un puntaje entre 50 y 60 en un instrumento que mide el interés generativo indicaron mayor satisfacción con las actividades diarias realizadas.

La variable interés generativo medida en cuidadores de adultos mayores mediante la Escala de Generatividad de Loyola (EGL) se clasificaría como variable intervalar al obtener el puntaje total de la prueba, el cual oscila de 0 a 60 puntos, donde altos puntajes indican un mayor interés generativo. Esto debido a que la prueba antes mencionada establece que la distancia entre cada uno de sus puntajes es la misma y el 0 no indica ausencia de interés generativo, sino solamente muestra el inicio de la evaluación.

4. En un centro de atención múltiple durante una evaluación cognitiva los niños que lograron recordar en un minuto 15 palabras de una lista de 30, también mostraron un vocabulario más extenso.

Las variables de razón son empleadas con menor frecuencia en el contexto psicológico. Un ejemplo podría ser el número de palabras recordadas en un minuto después de leer una lista de 30 palabras, de esta manera habrá participantes que recuerden $5,10,15$ palabras, o incluso quien no recuerde ninguna, lo que hablaría de la existencia de un cero absoluto.

\subsection{Clasificación por el grado de manipulación}

El punto central de la mayor parte de la investigación -sino es que toda- toma en cuenta el estudio de variables, esto independientemente de la disciplina científica. Sin embargo, a pesar del valor académico de la actividad científica que confiere la formación profesional de pregrado (licenciatura), todavía es difícil identificar la amplia categorización de las variables, concretamente en lo que se refiere al grado de manipulación. Por ello, es indispensable conocer su clasificación, donde se identifican las variables independientes (VI), variables dependientes (VD), las de tipo atributivas, activas y extrañas. En la figura 5 se aprecia la clasificación de las variables por la noción de producir o recibir efectos. 


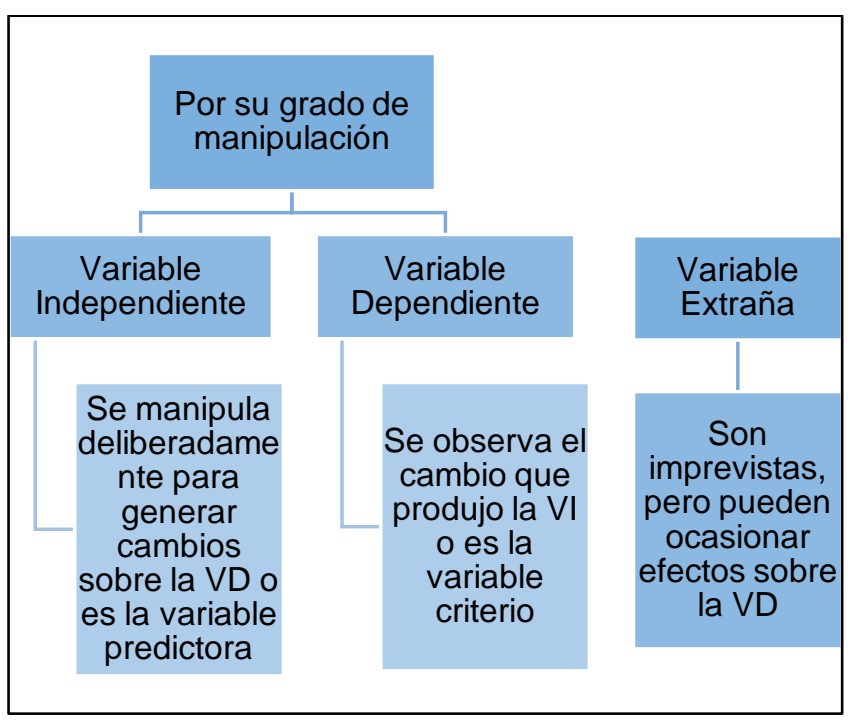

Fig. 5. Clasificación de las variables por su grado de manipulación

Variable independiente (VI) y Variable dependiente (VD). En los diseños experimentales, la VI se manipula deliberadamente para generar cambios o efectos sobre la VD, así, en esta última se observa el cambio o resultado que produjo la VI. Es importante, destacar que el rol de las variables (p.ej., independiente o dependiente) está en función de los objetivos de investigación, es decir, puede suceder que la VI de una investigación funja como la VD en otro estudio y viceversa. En cualquier caso, tomando en cuenta la simbología en las matemáticas, se dice que la $\mathrm{VI}$ es $\mathrm{X}$ y la VD es $\mathrm{Y}$, por tanto, se expresa con la siguiente ecuación $Y=f(X)$, es decir que, $Y$ está en función de X. En los siguientes ejemplos se pueden observar la VI y la VD.

1. La investigadora privó de sueño a sus pacientes para evaluar el efecto de dicha privación sobre la concentración en una tarea.

En el ejemplo anterior la privación de sueño es una $\mathrm{VI}$, debido a que la investigadora intencionalmente la manipuló para ver el efecto sobre la VD (concentración en una tarea). No obstante, la misma VD (concentración en una tarea), puede convertirse en $\mathrm{VI}$, como se puede apreciar en el siguiente ejemplo:

2. Un investigador manipuló la concentración en una tarea para evaluar su efecto sobre la precisión en el seguimiento de una instrucción verbal.

Como se observa, la variable concentración en una tarea en este caso fungía como VI (se manipuló), mientras que la VD fue el seguimiento de una instrucción verbal.

Lo anteriormente descrito se enmarca en los diseños experimentales que se caracterizan por la manipulación de la VI. Sin embargo, en los diseños no experimentales caracterizados por la ausencia de manipulación de variables, se puede determinar la $\mathrm{VI}$ a través de análisis estadísticos con alcance explicativo (de causalidad), no obstante, ésta se conoce como variable predictora, mientras que la VD se identifica como variable criterio. En los siguientes ejemplos se observan variables predictoras y variables criterio.

3. Un grupo de investigadores determinó que la influencia de pares en estudiantes mujeres de secundaria tuvo un efecto del $72 \%$ sobre la insatisfacción corporal $(r=.85 ; R 2=.72, p<.05)$. En el ejemplo anterior, la variable influencia de pares es una variable predictora, es decir, estadísticamente se determina como una VI, mientras que la insatisfacción corporal (variable criterio) es una VD.

Ahora bien, como ya se mencionó la VI se manipula para evaluar los efectos sobre otras variables de estudio, sin embargo, existen otras variables que de forma aleatoria pueden producir efectos, éstas son las variables extrañas, mismas que a continuación se describen.

Las variables extrañas como su nombre lo indica se tratan de atributos o características ajenas o no relacionadas con el propósito del estudio (extraña), regularmente, estas son imprevistas, pero pueden ocasionar efectos sobre la VD, de este modo, este tipo de variables podrían dar lugar a lo que se denominan resultados espurios, o cometer los errores tipo I o tipo II, esto es, reportar que hubo efecto cuando en realidad no lo hubo y viceversa. En los diseños experimentales es posible controlar la aparición de variables extrañas, ya que se cuida el contexto y la situación experimental, no obstante, en los diseños no experimentales, el control se realiza tanto como la situación lo permite. La variable extraña no se puede medir (porque ya no sería extraña), pero se debe reportar, para tomar con precaución los hallazgos de investigación. A continuación, se presenta un ejemplo de variable extraña.

4. Durante una intervención cognitivo-conductual para desarrollar la habilidad matemática en niños de primaria con problemas de repetición de ciclo escolar, los investigadores reportaron un efecto positivo de la intervención. Sin embargo, sucedió que, durante el programa de intervención, como parte de una actividad extra-clase, los niños recibieron lecciones gratuitas sobre el programa de aprendizaje de las matemáticas Kumon.

En el ejemplo anterior, los resultados reportados sobre la efectividad de la intervención pueden estar alterados por la variable extraña (lecciones Kumon), por lo que al 
enterarse los investigadores deben reportar dicha variable para tomar con precaución sus hallazgos.

\subsubsection{Clasificación de las variables independientes}

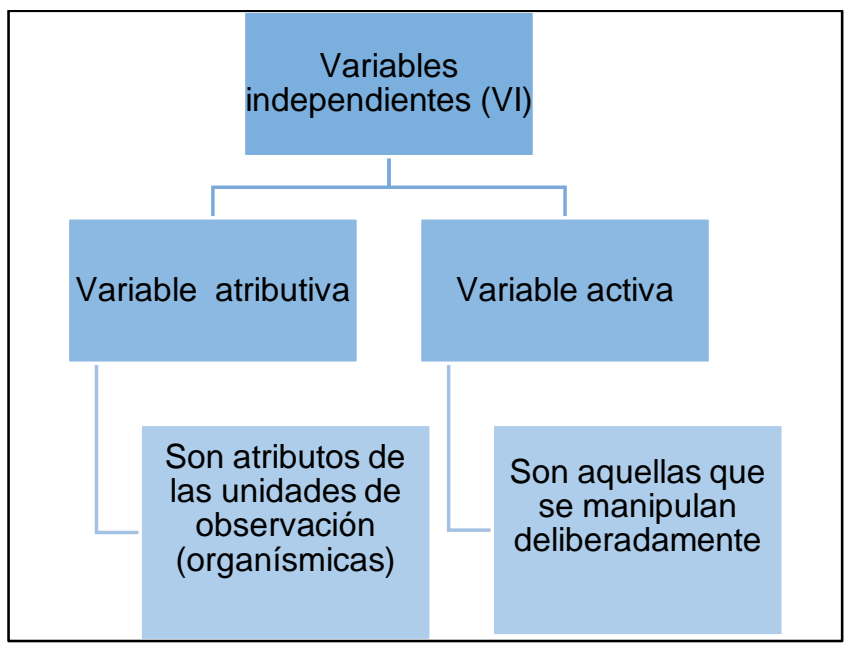

Fig. 6. Clasificación de las variables independientes

De manera general, las VI pueden ser atributivas o activas, a su vez estas últimas se pueden clasificar en fijas o aleatorias. A continuación, se describen cada una de ellas.

Las variables atributivas como su nombre lo dice son atributos de las personas, objetos o animales, en el caso concreto de personas y animales, también se les conoce como organísmicas, porque son inherentes al organismo.

1. Las estudiantes mujeres reportaron habilidades sociales deficientes, en comparación con los hombres.

La variable sexo (hombre o mujer) es una variable atributiva, que de ninguna manera se puede manipular, por lo que su comparación es ideal (mujeres vs. hombres), respecto a las habilidades sociales.

\section{Los pacientes de estatura baja indicaron llevar a cabo mayor número de dietas restrictivas.}

La variable estatura es atributiva, esta no se puede manipular, pero su clasificación es ideal (baja, media o alta) al interior de una muestra.

Las variables activas son aquellas que se manipulan deliberadamente, es decir el investigador modifica intencionalmente la variable para evaluar el efecto que produce en otra(s) variable(s), estas pueden ser, aleatoria o fija (controlada). En los siguientes ejemplos se observan variables activas.
3. Las ratas (sujetos experimentales) recibieron alimento enriquecido con vitaminas y reportaron mejor orientación espacial en el laberinto.

La variable "alimento enriquecido" es VI activa-fija, ya que a todos los sujetos de experimentación deliberadamente se les suministró el mismo tipo de alimento.

4. En las ratas con sobrepeso que se privaron de agua, se encontró mejor tasa de respuesta de palanqueo en comparación con aquellas que se privaron de alimento o de sueño. Cabe mencionar que el tipo de privación fue asignado aleatoriamente.

En el ejemplo anterior, el investigador manipuló activamente tres tipos de privación (agua, alimento o sueño) y para fortalecer la validez interna del experimento asignó aleatoriamente el tipo de privación al que se sometería a cada grupo. Así, la variable privación es una $\mathrm{VI}$ activa-aleatoria.

Como se ha revisado, existen diversas formas de clasificar a las variables de acuerdo con diferentes criterios. Resulta importante tomar en cuenta los objetivos y alcances de la investigación para identificar las propiedades de las variables en estudio, así como, sus definiciones como se verá en el siguiente apartado.

\section{Definición conceptual y operacional de una variable}

Los constructos o variables deben ser claramente definidos para poder ser observados, medidos 0 evaluados; se pueden definir en dos sentidos a) con el uso de otras palabras como lo hace el diccionario o b) por acciones, comportamientos 0 indicadores que los expresan.

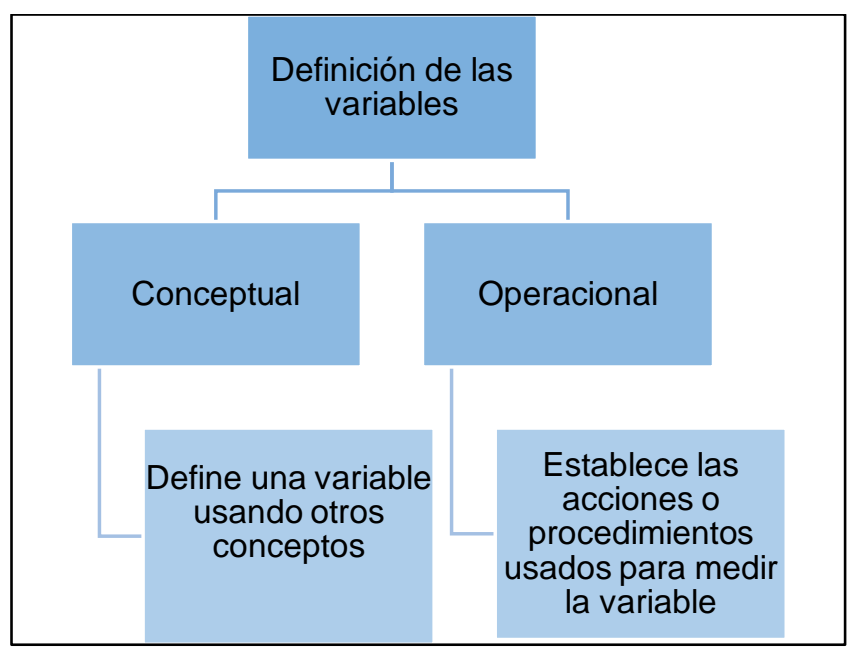

Fig. 7. Tipos de definición de las variables 
Una definición conceptual o constitutiva define una variable usando otros conceptos. ${ }^{1}$ Es decir, es aquella que describe a la variable a través de palabras derivadas de investigación, teorías o modelos. Generalmente la hace un autor ajeno al investigador y se retoma del marco teórico.

Torgenson $^{12}$ indica que todas las variables deben poseer una definición conceptual para tener un significado que pueda ser usado en teorías, y ser científicamente útiles. Así, es importante tener una definición conceptual para poderla retomar en otras investigaciones.

Algunos ejemplos de definiciones conceptuales se presentan a continuación:

1. Generatividad: Es el interés por las generaciones futuras con el objetivo de dejar un legado que sobreviva mediante actividades como el cuidado de otros, el compromiso social, la crianza, el compartir conocimientos y/o la participación cívica y política. ${ }^{13}$

En esta definición se parafrasea a otros autores que han investigado la variable, para definir la Generatividad.

2. Calidad de vida: "Es la percepción de los individuos de su posición en la vida en el contexto de su cultura y sistema de valores, en la cual ellos viven y en relación a sus metas, expectativas, estándares e intereses." ${ }^{.14}$ (p.551)

En este ejemplo, se toma como cita textual la definición que otros autores han dado de la variable Calidad de Vida para definirla conceptualmente.

Por otra parte, se sabe que no hay investigación científica sin observación o experimentación y esta no es posible sin instrucciones claras y específicas de qué, cómo y cuándo observar, medir o manipular.

Por lo tanto, "una variable debe traducirse en formas concretas de observación o manipulación ${ }^{15 "}$ (p.71). Las variables deben describirse para poder estudiarse de manera empírica y para ello existen las definiciones operacionales, que indican exactamente el significado del constructo en la investigación en curso.

Una definición operacional, observacional o conductual, define una variable estableciendo las acciones específicas o procedimientos usados para medirla ${ }^{16}$, es decir, asigna significado a una variable al especificar las actividades $u$ operaciones necesarias para que el investigador pueda realizar la experimentación y la medición. ${ }^{1}$ Implica algo así como un compendio de instrucciones abreviado.
Las definiciones operacionales tienen dos principales objetivos, el primero es la réplica, es decir que, si alguien más en el futuro desea repetir el estudio, debe saber exactamente como se midieron o manipularon las variables; el segundo objetivo, es hacer posible la verificación y el enriquecimiento del significado de una variable, dando claridad y acotando para mejorar la comprensión.

De manera general, existen dos tipos de definiciones operacionales: las de experimentación o proceso y las de medición. ${ }^{1,10}$ La definición operacional experimental o de proceso señala los detalles (operaciones de la manipulación de una variable por parte del investigador). Por ejemplo, el reforzamiento puede definirse operacionalmente al precisar los detalles de cómo los sujetos serán reforzados y no reforzados o cómo se llevó a cabo la manipulación. A continuación, se presenta un ejemplo de definición operacional de experimentación o proceso:

3. Variabilidad de la frecuencia cardiaca definida operacionalmente como el registro psicofisiológico realizado mediante el decodificador multimodal Procomp Infiniti, que es un dispositivo que cuenta con ocho canales de medición que monitorean un gran rango de señales fisiológicas. Para la transmisión de dichas señales se utiliza un cable de fibra óptica que conecta a la unidad decodificadora y la unidad de interfaz rápida USB a un escritorio que ejecute el software Use3 Physiolab con Windows; el cual proporciona retroalimentación, recoge datos, informes impresos y exporta archivos de bases de datos. Así la variabilidad de la frecuencia cardiaca se evaluó mediante los canales diferenciales de Electrocardiograma (ECG) para obtener las ondas de intervalo $R R$ (Variaciones en el intervalo de tiempo entre latido y latido que se calcula entre la diferencia de una onda $R$ a otra onda $R$ ) en tres momentos, durante 3 minutos de reposo, durante 3 minutos de evocación de estrés (cuando se dio la indicación a los participantes que evocaran el evento más estresante que hayan experimentado a lo largo de su vida) y durante 3 minutos de recuperación (cuando se les dio la instrucción de que dejarán de pensar en el evento). Posteriormente los datos fueron limpiados a través del software QRStool, $y$ se pasaron al procesador KUBIOS para tener los datos en numéricos.

Como se observa en el ejemplo, la definición operacional de experimentación o proceso es exclusiva de diseños 
experimentales y requiere la descripción detallada de la manipulación de la VI.

En contraste, como su nombre lo indica, las definiciones operacionales de medición describen cómo será medida la variable a través de indicadores, instrumentos o conductas.

Los indicadores, son los elementos que componen a una variable, a partir de ellos se puede inferir la presencia, ausencia o intensidad de esta, como lo muestra el siguiente ejemplo:

4. Se definió operacionalmente a la variable nivel socioeconómico a través de los siguientes indicadores: ingreso en salarios mínimos, número de bienes muebles, número de bienes inmuebles, clasificación de la zona en la que vive (residencial, habitacional, vulnerable, muy vulnerable) número de focos en su casa, número de aparatos electrodomésticos, tipo de propiedad de la vivienda (propia, rentada o de familiares), tipo de vivienda (choza, departamento, casa), número de plantas de la casa (una planta, dos o más plantas), servicios de la casa (piso, drenaje, luz, agua, gas, teléfono, tv por cable, servicio de internet), etc.

Así, en esta investigación, se puede inferir que una persona tiene alto nivel socioeconómico si tiene un ingreso mayor a 30 salarios mínimos, cuenta con bienes muebles o inmuebles, vive en una zona residencial, tiene más de 15 focos en casa, cuenta con múltiples aparatos electrodomésticos, y vive en una casa propia de varias plantas con todos los servicios. Por el contrario, se puede inferir que una persona tiene un bajo nivel socioeconómico si tiene un ingreso menor a un salario mínimo, no cuenta con bienes muebles o inmuebles, vive en una zona de alta vulnerabilidad, tiene menos de 5 focos en casa, solo cuenta con un aparato electrodoméstico, y vive en una casa de familiares de una sola planta sin servicios.

Los instrumentos son otras formas indirectas de evaluación que permiten realizar definiciones operacionales de medición, aunque para ello deben contener forzosamente los análisis psicométricos que les avalen (validez y confiabilidad para la población de estudio así como utilidad práctica, como mínimo), como se muestra a continuación:

5. La variable amor definida operacionalmente como el puntaje obtenido en la Escala Triangular del Amor de Sternberg ${ }^{17}$ validada para población mexicana por Mazadiego y Garcés $^{18}$, y que cuenta con un Alpha total de .70. El instrumento consta de 45 ítems, 15 para cada uno de los tres componentes: Pasión, intimidad y compromiso. Donde: Intimidad es el nivel de autodivulgación y cercanía con la pareja, pasión es el arrebato emocional hacia el otro y compromiso es el establecimiento de la relación a largo plazo y el deseo de permanecer en ella.

En este estudio, a mayor puntuación en la escala más amor y a menor puntaje en la escala menos amor.

La definición operacional de medición conductual u observacional describe las conductas que conforman el constructo o variable. Es decir, se detallan las acciones que el sujeto debe seguir para poder ser evaluado. Regularmente estas se documentan a través de listas de chequeo o registros observacionales. Aquí un ejemplo:

6. La variable violencia física en la pareja es definida operacionalmente como aquellos golpes, jaloneos o pellizcos llevados a cabo por un miembro de la pareja hacia el otro en el periodo de observación comprendido por 24 horas, durante una semana. Incluyendo dentro de la categoría de golpes, las bofetadas, los puñetazos, los golpes con algún objeto, con la mano abierta o con alguna parte del cuerpo hacia el otro. Las conductas serán recabadas en una hoja de registro donde se contabilizará la frecuencia de ocurrencia por semana.

Así en el ejemplo anterior, a mayor frecuencia, mayor violencia física y a menor frecuencia menor violencia.

\section{Evaluación de las variables}

Como se ha revisado, una de las características esenciales de las variables es que son susceptibles de medición. En el ámbito psicológico se cuenta con una amplia variedad de instrumentos que permiten registrar los datos reportados por los participantes. De acuerdo con García, Ramírez y Ávila ${ }^{9}$ se identifican 4 grandes grupos de instrumentos que a continuación se describen:

\section{Registros observacionales}

1.1.De frecuencia, se registra la conducta a evaluar cada vez que el participante la ejecute, con lo que se medirá la frecuencia de aparición del evento.

1.2. De duración, se mide el momento de aparición de la conducta a evaluar, así como el momento de terminación, con lo cual se medirá la ocurrencia del evento.

1.3. De bloques, se seleccionan periodos de observación específicos en los cuales se registra si la conducta a evaluar se presenta o no. 
2. Guías de entrevista

2.1. Estructurada, se apega a una serie de preguntas previamente establecidas, las cuáles se formulan a todos y cada uno de los participantes en el mismo orden, sin posibilidades de modificación.

2.2. Semiestructurada, tiene fundamento en una serie de preguntas previamente elaboradas que sirven como guía; no obstante, el investigador puede adaptarlas según las características de los participantes 0 de las respuestas obtenidas en el momento.

2.3. No estructurada, se fundamenta en un esquema general de temas que se deben abordar a lo largo de la entrevista, no obstante, no existen preguntas formuladas previamente de manera que el investigador es libre de formular los cuestionamientos como crea que es más conveniente para cada participante.

3. Escalas de actitudes, miden la intensidad de las actitudes hacia cierto evento, circunstancia o tema.

3.1. Escala Likert, es una escala ordinal que consiste en un conjunto de afirmaciones o juicios que miden cuánto más o menos desfavorable es una actitud en tres, cinco o siete categorías u opciones de respuesta.

3.2. Diferencial semántico, se establecen una serie de pares de adjetivos extremos que sirven para calificar el objeto de una actitud, ante los cuales se pide la reacción del participante y lo ubica en una categoría por cada par.

4. Pruebas estandarizadas, miden a las variables de manera más precisa. Cada prueba estandarizada tiene sus propios procedimientos de aplicación, codificación, calificación e interpretación; además han sido sometidos a procesos para calcular sus propiedades psicométricas tales como confiabilidad y validez, son estandarizados ya que cuentan con normas y parámetros de calificación.

Por ejemplo, una variable cuantitativa se puede medir a través de un registro.

1. Una psicóloga clínica, registró la conducta disruptiva de un niño con Trastorno con Déficit de Atención con Hiperactividad durante una hora los cinco días de la semana.

En el ejemplo anterior, fue necesario en primer lugar operacionalizar la variable (conducta disruptiva), posteriormente, la psicóloga determinó con base en las propiedades de la variable que un registro de frecuencia de conducta por sesión y por periodo establecido, era el medio más adecuado para medir y observarla.

Las variables pueden ser evaluadas a través de entrevistas, como en el siguiente ejemplo:
2. Se diseñó y aplicó una guía de entrevista semiestructurada para familiares de pacientes con influenza $A(H 1 N 1)$. La guía se sometió al proceso de validación por jueces expertos y a un proceso de piloteo.

La guía de entrevista cara a cara se enfocó en medir tres áreas:

a) Datos generales del entrevistado a través de los siguientes indicadores: sexo, edad, escolaridad y ocupación (4 preguntas).

b) Conocimientos e información sobre la influenza $A(H 1 N 1)$, que se midió a través de los siguientes indicadores: conocimientos previos de medidas de prevención, medidas de prevención empleadas durante la contingencia, recomendaciones para prevención de nuevas contingencias de salud e intención conductual de apego a medidas preventivas (10 preguntas).

c) Impacto psicosocial de la epidemia de influenza A(H1N1) que se midió a través de los siguientes indicadores: Reacción emocional a la alerta sanitaria (miedo, enojo, angustia, incertidumbre, etcétera), reacción emocional al padecimiento del familiar, percepción de apoyo por parte de sus redes, relación médico-familiar/paciente, nivel de alteración psicológica (Distress) experimentada durante la contingencia y nivel de desgaste emocional debido a la enfermedad del familiar (10 preguntas).

En el ejemplo anterior, fue necesario en primer lugar operacionalizar la variable (Impacto psicosocial de la epidemia de influenza $A(\mathrm{H} 1 \mathrm{~N} 1)$ ), posteriormente, con base en la definición elaborar la guía de entrevista y finalmente someterla a una evaluación previa para garantizar que midiera los indicadores objetivo.

Las variables cuantitativas también se pueden medir a través de escalas de actitud.

3. Se utilizó el Cuestionario de Actitudes hacia el Aborto (CAA) validada por Marván, OrihuelaCortés y Río. ${ }^{19}$ Se trata de una escala Likert de 5 puntos (1-completo desacuerdo, 5-completo acuerdo), con 23 reactivos divididos en tres factores que explican el 48,2\% de la varianza: (a) "pro vida", cuyos reactivos reflejan actitudes negativas hacia el aborto, considerándolo inhumano e injustificado; (b) "pro elección", cuyos reactivos reflejan el reconocimiento del derecho de las mujeres a abortar; $y$ (c) "derechos reproductivos", cuyos reactivos se refieren a implicaciones sobre los derechos reproductivos al no estar legalizado el aborto. La confiabilidad fue 
la siguiente: "Pro vida": 0,89, "Pro elección": 0,91 $y$ "Derechos reproductivos": 0,73.

Como se puede observar en el ejemplo anterior, las escalas de actitud a pesar de no tener puntos de corte si deben reportar validez y confiabilidad.

Las variables cuantitativas también se pueden medir a través de pruebas estandarizadas.

4. El equipo de investigadores realizó una evaluación para determinar el efecto de la autoestima sobre la insatisfacción corporal, la medición se realizó con una batería de pruebas estandarizados en la que incluyeron: a) Escala de Autoestima de Rosenberg que tiene el propósito de evaluar la percepción global del individuo respecto a la valía de sí mismo (auto-respeto y auto-aceptación); el rango de puntuación va de 10 a 40 estableciéndose tres niveles de puntuación 30-40 que indica autoestima elevada; 26-29 supone una autoestima media; $y<25$ es indicativa de baja autoestima ${ }^{20}$; b) Cuestionario de Imagen Corporal que evalúa la preocupación e inconformidad con la forma corporal (peso y figura) que experimenta una persona; el rango de puntuación va de 10 a 60 para el factor de preocupación por el peso y la figura y de 11 a 66 para el fator de insatisfacción corporal. El rango de puntuación total va de 34 a 204; en donde una mayor puntuación, indica mayor insatisfacción corporal. $^{21}$

En el ejemplo anterior, la VI (predictora) es la autoestima y la VD (criterio) es la insatisfacción corporal, posteriormente el equipo de investigadores realizará el análisis estadístico correspondiente para alcanzar su objetivo. Así, las pruebas estandarizadas son un medio valioso para medir las variables.

A continuación, se muestra un ejemplo de una variable que puede ser evaluada por más de un tipo de los instrumentos ya descritos; la decisión sobre qué instrumento emplear será tomada por el investigador de acuerdo con los objetivos y alcances de su investigación.

\section{VARIABLE: Sobrecarga del cuidador}

\section{INSTRUMENTO A UTILIZAR: PRUEBA ESTANDARIZADA}

El investigador ha decidido emplear la Escala de carga del cuidador de Zarit que evalúa la carga de los cuidadores primarios a partir de identificar los sentimientos habituales que experimentan quienes cuidan a otra persona. Se compone de 22 reactivos tipo Likert con cinco opciones de respuesta: 0 "nunca", 1 "rara vez", 2 "algunas veces", 3 "bastantes veces y 4 "siempre".

El rango del puntaje total va de 0 a 88 puntos, donde altos puntajes indican una elevada carga al cuidador. Los puntos de corte de dicha escala son:

$\leq 46$ puntos no hay sobrecarga

47 a 55 puntos hay sobrecarga leve

$\geq 56$ puntos hay sobrecarga intensa

\section{INSTRUMENTO A UTILIZAR: REGISTRO} OBSERVACIONAL DE FRECUENCIA

El investigador ha establecido previamente que las conductas gritar e ignorar, son signos evidentes de que el cuidador ha desarrollado sobrecarga. Cada una de estas conductas fue definida de la siguiente manera

Gritar: El cuidador emite un sonido lo suficientemente fuerte para causar expresión o efecto de aversión y que contiene una instrucción o comentario que expresa desaprobación. Por ejemplo: ¡Que mal lo hizo! ¡No se levante de la cama!, ¡No se retire del comedor!

Ignorar: Ante una petición verbal del paciente, el cuidador no le contesta antes de diez segundos o no establece contacto visual asintiendo con la cabeza, no se sigue de la acción solicitada o la explicación amable de por qué no es posible o conveniente.

El investigador establece períodos de observación de 10 minutos en la interacción cuidador-paciente y registrará durante esos 10 minutos cuántas veces se presentó la conducta de gritar y cuántas veces se presentó la conducta de ignorar.

\section{INSTRUMENTO A UTILIZAR: GUÍAS DE ENTREVISTA}

El investigador ha decidido emplear la Entrevista no estructurada de Carga del cuidador $^{22}$ para evaluar la sobrecarga. En dicha entrevista se han establecido previamente los tópicos a tratar:

1. Aspectos sociodemográficos tanto de la persona cuidada como de la persona que presta los cuidados.

2. Características del cuidado evaluando el tiempo que el cuidador lleva cuidando al paciente, así como la calidad percibida de su relación antes y después de que el paciente requiriera recibir cuidados especiales.

\section{Consideraciones finales}

Algunos aspectos a tomar en cuenta acerca de las variables se mencionan a continuación:

Las variables son atributos cuya variación es susceptible de medirse; en psicología y en otras ciencias sociales y de la salud, dichos atributos son parte cardinal en la actividad científica, debido a que se observan, miden y reportan. 
Existen al menos cinco rubros para clasificar las variables: I) por el número de sus valores; II) por su naturaleza (cuantitativa o cualitativa); III) por el nivel de medición (nominal, ordinal, intervalar y razón); IV) por el grado de manipulación (dependiente e independiente); V) por su definición (conceptual y operacional).

Respecto a esta última clasificación, se debe resaltar que, en un trabajo de investigación, todas las variables deben tener tanto definición conceptual como operacional y ser congruentes entre sí, lo que evitará la ambigüedad y potencializará la comparación con datos de otros estudios. No obstante, ninguna definición operacional puede expresar toda la riqueza de las variables, por lo tanto, las variables medidas por los investigadores siempre tienen un significado limitado y específico y estarán en función de los objetivos de investigación.

Finalmente, la decisión sobre qué instrumento emplear para medir una variable será tomada por el investigador de acuerdo con los propósitos y alcances de su investigación

\section{Referencias}

[1] Kerlinger F, Lee H. Investigación del comportamiento. México: McGraw-Hill; 2002.

[2] Real Academia Española de la Lengua. Diccionario de la lengua española. $23^{\mathrm{a}}$ ed. España: Autor; 2014

[3] Moulines C. Fundamentos de Filosofía de la Ciencia. Barcelona: Alianza Editorial; 1997.

[4] American Psychiatric Association. DSM-5: Manual diagnóstico y estadístico de trastornos mentales. $5^{\text {a }}$ ed. Madrid: Editorial Médica Panamericana; 2014

[5] García B. Manual de métodos de investigación para las ciencias sociales. Un enfoque de enseñanza basado en proyectos. México: Manual Moderno; 2009

[6] Ritchey F. Estadística para las Ciencias Sociales. México: Mc Graw Hill; 2008.

[7] Cruz C, Olivares S, González M. Metodología de la investigación. México: Grupo Editorial Patria; 2010.

[8] Nuñez I. Las variables: estructura y función en la hipótesis. Inves Educ. 2007[acceso: 08/05-21]; 11(20):163-179. Disponible en: http://revistasinvestigacion.unmsm.edu.pe/index.php/educa/article/vie wFile/4785/3857

[9] García B, Márquez L, Ávila J. Planeación y desarrollo del proyecto de investigación. En: García B. editor. Manual de métodos de investigación para las ciencias sociales. Un enfoque de enseñanza basado en proyectos. México: Manual Moderno; 2009. p. 3-47

[10] González-Celis ALM. Las variables en Psicología: su clasificación, definición y medición. En Chávez B., Tron R. Coordinadores. Análisis descriptivo en Psicología. Aspectos metodológicos y estadísticos. México: UNAM, FESI; 2012. p. 19-67.

[11] Cohen R, Swerdlik M. Pruebas psicológicas: introducción a las pruebas y la medición. México: McGraw-Hill; 2006.
[12] Torgerson W. Theory and methods of scaling. Melbourne: Krieger; 1985.

[13] Villar F, López O, Celdrán M. La generatividad en la vejez y su relación con el bienestar: ¿Quién más contribuye es quien más se beneficia? Anales Psic. 2013[acceso 13/05/21]; 29(3): 897-906. Disponible

https://scielo.isciii.es/scielo.php?script=sci_arttext\&pid=S021297282013000300029

[14] Harper A, Power M. Development of the World Health Organization WHOQOL-Bref quality of life assessment. Psych Medic. 1998[acceso: 12/05/21]; 28: 551-558. Disponible en: https://pubmed.ncbi.nlm.nih.gov/9626712/

[15] Cozby P. Métodos de investigación del comportamiento. $8^{\mathrm{a}}$ ed. California: Cal State Univer; 2005

[16] Coon D, Miterer J. Introducción a la psicología: el acceso a la mente y la conducta. México: Cengage; 2017

[17] Sternberg R. Triangular theory of love. Psych Rev. 1986[acceso: 13/05/2021]; 93(2); 119-135. Disponible en: https://www.semanticscholar.org/paper/A-triangular-theory-of-love.Sternberg/3ece4ab9b51b22105e654e2c587d2c4fbd19f59d

[18] Mazadiego T, Garcés J. El amor medido por la Escala Triangular de Sternberg. Psicolatina. 2011[acceso: 13/05/21];22(1): 1-10. Disponible en: http://psicolatina.org/22/seccion1/amor.pdf

[19] Marván M, Orihuela-Cortés F, Río AÁD. Actitudes hacia la interrupción voluntaria del embarazo en jóvenes mexicanos, y su opinión acerca del aborto inseguro como problema de salud pública. Cad Saú Púb. 2018[acceso 08/05/21]; 30(10); 1-8. Disponible en: https://doi.org/10.1590/0102-311X00192717

[20] González-Forteza C, Andrade P, Jiménez T. Recursos psicológicos con el estrés cotidiano en una muestra de adolescentes mexicanos. Salud Ment. 1997[acceso: 13/05/21]; 20(1): 27-35. Dsiponible en: http://www.revistasaludmental.mx/index.php/salud_mental/article/vie w/639

[21] Vázquez R., Galán JC, López X, Alvarez G, Mancilla J M, Caballero A, Unikel C. Validez del Body Shape Questionnaire (BSQ) en mujeres mexicanas. Rev Mex Tras Alimen. 2011(acceso 12/05(21]; 2(1): 4252. http://www.scielo.org.mx/scielo.php?script=sci_arttext\&pid=S200715232011000100005

[22] Zarit S, Zarit J. The Memory and behavior Problem Checklist and the Burden Interview. Technical Report. Pensilvania; Pennsilvania State University; 1983. 